1. Arteche E, Ostiz S, Miranda L, Caballero P, Jiménez López de Oñate G. Tromboflebitis séptica de la vena porta (pileflebitis): diagnóstico y manejo a propósito de tres casos. An Sist Navar 2005; 28 (3): 417-20.

2. Balthazar EJ, Gollapudi P. Septic Thrombophlebitis of the Mesenteric and Portal Veins: CT Imaging. J Computer Assist Tomography 2000; 24: 755-60.

3. Plemmons RM, Dooley DP, Lonfield RN. Septic thrombophlebitis of the portal vein (pylephlebitis): Diagnosis and management in the modern era. Clin Infect Dis 1995; 21: 1114-20.

4. Pelsang RE, Johlin F, Dada R, Bogdanowicz M, Schweiger GD. Management of Suppurative Pylephlebitis by Percutaneous Drainage: Placing a Drainage Catheter into the Portal Vein. Am J Gastroenterol 2001; 96 : 3192-4.

\section{Neurofibrosarcoma con metástasis pulmonares en paciente con neurofibromatosis tipo I}

\section{Sr. Director:}

La neurofibromatosis tipo 1 (NF 1) afecta a 1 de cada 3.000 personas. Aproximadamente la mitad de los casos son familiares. Tiene una herencia autosómica dominante pero entre un 30-50\% de los casos son debidos a mutaciones espontáneas. Su pronóstico se halla ensombrecido por la malignización de los tumores y las complicaciones derivadas de los trastornos circulatorios y la hipertensión en relación con la aparición de feocromocitomas. Presentamos un paciente con neurofibromatosis tipo 1 donde la presencia de una degeneración sarcomatosa de uno de sus neurofibromas condicionó su evolución desfavorable.

Varón de 19 años diagnosticado neurofibromatosis tipo 1 (NF-1) a los 3 meses de edad. No hay antecedentes familiares de neurofibromatosis. Consulta por dolor en extremidad inferior izquierda de 2 meses de evolución que ha empeorado a lo largo del tiempo, asociándose la aparición de debilidad en la marcha. En la exploración se observan pecas axilares, manchas café con leche y neurofibromas lumbares, cara anterior de extremidades superiores y en muslo izquierdo. No hay alteración intelectual ni oftalmológica. Cifras de tensión arterial normales. Debilidad para flexoextensión de rodilla y pie izquierdos con arreflexia aquílea e hipoalgesia en cara antero-externa de pierna izquierda con signo de Tinel positivo. En el EMG se observan signos denervativos agudos (fibrilaciones y ondas positivas) en musculatura dependiente del nervio ciático izquierdo, sobre todo en nervio poplíteo interno en probable relación con compresión en muslo por la presencia de neurofibroma de gran tamaño. Se realiza resonancia magnética lumbar y de muslo izquierdo evidenciándose numerosos neurofibromas foraminales y paravertebrales sin compromiso raquídeo y uno de mayor diámetro $(7 \mathrm{~cm})$ en región posterior del muslo en relación con el trayecto del nervio ciático y sin compromiso de vasos femoro-poplíteos (Fig. 1).

Al realizar exéresis de la tumoración que se extendía desde hueco poplíteo hasta pliegue glúteo izquierdo se evidenció un neurosarcoma. En radiografía de tórax se observaba opacidad de hemitórax derecho y masa en hemitórax izquierdo y en la tomografía computarizada toracoabdominal presentaba una gran tumoración extrapulmonar que ocupa la práctica totalidad del hemitórax derecho con atelectasia del pulmón subyacente y desplazamiento mediastínico contralateral, otra tumoración a nivel apical izquierdo y numerosos nódulos pulmonares (Fig. 1) compatibles con metástasis con lo que se clasificó de estadio IIIB de Enneking. Se decidió amputación de extremidad inferior izquier-

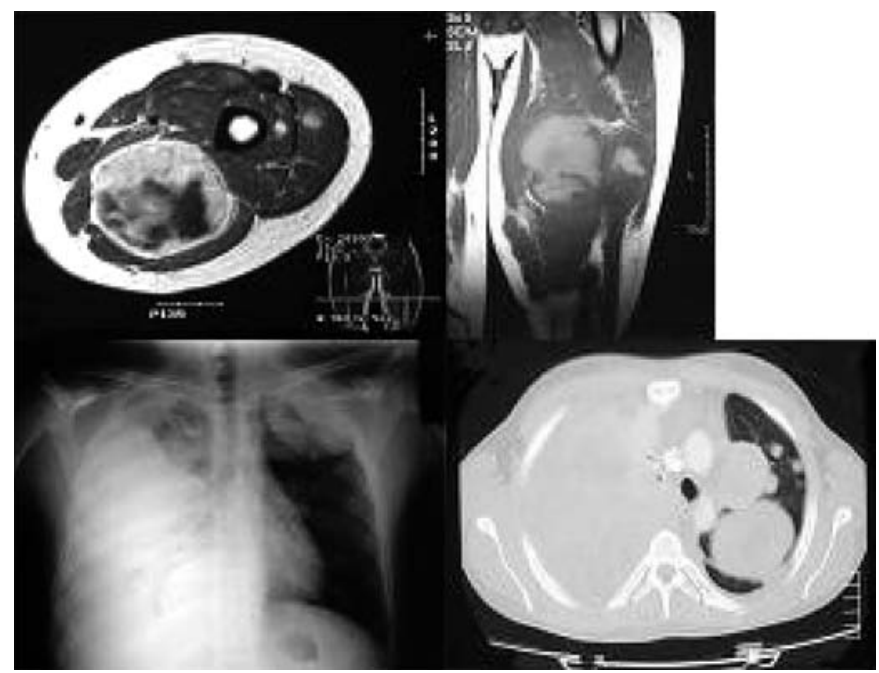

Fig. 1.

da y tres ciclos de quimioterapia con ifosfamida y adriamicina con ausencia de remisión siendo éxitus.

La neurofibromatosis tipo I o enfermedad de von Recklinghausen (NF1) es la más frecuente de las neurofibromatosis (aproximadamente el $85 \%$ de los casos). Hay una afectación en el cromosoma 17 (17q11.2). Este gen codifica una proteína, la neurofibromina que actúa como supresor tumoral a través de una regulación negativa del Ras (1). La penetrancia es completa pero la expresividad clínica es muy variable. Los criterios diagnósticos propuestos por la Conferencia Consenso en 1987 y revisados en 1997 se basan en hallazgos clínicos específicos. De acuerdo a estos criterios deben estar presentes al menos dos de los siguientes hallazgos clínicos para diagnosticar una NF1: a) seis o más manchas café con leche de diámetro mayor a $5 \mathrm{~mm}$ antes de la pubertad y $>15 \mathrm{~mm}$ después de ella; b) dos o más neurofibromas de cualquier tipo o uno plexiforme; c) moteado en región axilar o inguinal; d) glioma óptico; e) dos o más nódulos de Lisch (hamartomas del iris); f) una lesión ósea distintiva (displasia esfenoidal o adelgazamiento de la cortical de huesos largos, con pseudoartrosis o sin ella); y g) un familiar de primer grado con NF1, según los criterios anteriores. El orden típico de aparición de la clínica es: manchas café con leche, moteado axilar, nódulos de Lisch y neurofibromas. Los neurofibromas son tumores benignos compuestos por células de Schwann, fibroblastos y mastocitos. Existen tres tipos de neurofibromas: cutáneos, subcutáneos y plexiformes. Los neurofibromas plexiformes representan la principal causa de morbilidad, desfiguración y mortalidad en jóvenes con NF1. Estas lesiones involucran habitualmente a varios fascículos nerviosos y presentan una importante vascularización lo que hace extremadamente difícil, si no imposible su resección quirúrgica. Los pacientes con NF1 presentan mayor frecuencia de tumores tanto benignos como malignos. El riesgo de malignización en NF1 se estima en torno a un 4 a un $10 \%$ de los individuos afectos (2). Los neurofibromas plexiformes pueden sufrir transformación maligna a tumores de la vaina de nervios periféricos también llamados neurofibrosarcomas o schwanomas (3) que se desarrollan más tempranamente que en la población general (4). Estos tumores presentan mal pronóstico debido a su recurrencia (5) y/o aparición de metástasis posterior a su resección (6). En un paciente con NF1 hay que vigilar los cambios en el tamaño y la aparición de dolor en los neurofibromas. Estos cambios podrían deberse a una transformación maligna que origina un empeoramiento pronóstico (7). En nuestro caso la aparición de un neurofibrosarcoma con metástasis condicionó el fallecimiento del paciente. 


\section{R. Merchán Rodríguez, P. Cacabelos Pérez ${ }^{1}$, C. Delgado², M. Alañá García ${ }^{1}$}

Servicio de Medicina Interna. ${ }^{1}$ Servicio de Neurología. ${ }^{2}$ Servicio de Oncología. Hospital Clínico Universitario de Salamanca. Salamanca

1. Plon SE, Blazo M. Neurofibromatosis type 1 (von Recklinghausen's disease). Uptodate 2005. Disponible en URL: http://www.utdol.com

2. Kim DH, Murovic JA, Tiel RL, Mos G, Kline DG. A series of 397 peripheral neural sheath tumors: 30-year experience at Louisiana State University Health Sciences Center. J Neurosurg 2005; 102: 246-55.

3. Schorry EK, Crawford AH, Egelhoff JC, Lovell AM, Saal HM. Thoracic tumors in children with neurofibromatosis-1. Am J Med Genet 1997; 74: 533-7.

4. Ligneau B, Freyer G, Giraud S, Isaac S, Bombaron P, Espinouse D, et al. Neurosarcoma associated with neurofibromatosis 1 . A propos of a case and review of the literature. Presse Med 1997; 26: 458-63.

5. Topsakal C, Akdemir I, Tiftikci M, Ozercan I, Aydin Y. Malignant schwannoma of the sciatic nerve originating in a spinal plexiform neurofibroma associated with neurofibromatosis type 1. Neurol Med Chir (Tokyo) 2001; 41: 551-5.

6. Ramirez JA, Montesinos JJ. Case 3: Neurofibromatosis I with metastases from a thigh neurofibrosarcoma. Am J Roentgenol 1998; 171: 837, 841-2.

7. Kam JR, Helm TN. Neurofibromatosis. Disponible en en: http://www.emedicine.com/derm/topic287.htm.

\section{Acidosis láctica mediada por metformina}

\section{Sr. Director:}

La metformina es una biguanida ampliamente utilizada en el tratamiento de la diabetes. El UKPDS demostró que se asociaba a una menor ganancia de peso, menos hipoglucemias y un mejor control cardiovascular. Su indicación es en el paciente diabético y obeso aunque recientemente también se postula su uso en pacientes no obesos por ser al menos tan eficaz como en aquéllos (1). Su amplio uso hace que haya que conocer una serie de efectos adversos de potencial gravedad como la acidosis láctica. A continuación se describe un caso con evolución fatal.

Paciente mujer de 86 años sin alergias conocidas con buena capacidad funcional previa y antecedentes de colecistectomía, HTA, DM-2, cardiopatía isquémica en forma de angor hacía 1 año, ictus isquémico recuperado ad integrum hacía 6 años y una historia de diarreas de más de 5 años de evolución que habían sido estudiadas en múltiples ocasiones pero que únicamente se llegó al diagnóstico de diverticulosis. Seguía tratamiento con metformina $850 \mathrm{mg} / 8 \mathrm{~h}$, valsartan $160 \mathrm{mg} / 24 \mathrm{~h}$, hidroclorotiacida $12,5 \mathrm{mg} / 24 \mathrm{~h}$, clopidogrel $75 \mathrm{mg} / 24 \mathrm{~h}$ y nitratos tópicos.

Acude a nuestro centro por presentar desde hacía 3 días un cuadro de diarreas en número de 3-4 deposiciones líquidas nocturnas diferentes de las habituales según refería la familia con vómitos biliosos y dolor abdominal difuso. No referían ninguna otra sintomatología ni consumo de tóxicos, fármacos nuevos o sobredosificación de los habituales.

En la exploración destacaba un muy mal estado general con respiración de Kussmaul, taquicardia, tensión arterial de 200/90 e hipotermia. Saturación de $\mathrm{O}_{2} 95 \%$, palidez mucocutánea y agitación psicomotriz. El abdomen era doloroso difusamente a la palpación sin masas ni hepatoeseplenomegalias. No presentaba peri- tonismo pero sí escasos ruidos hidroaéreos con tacto rectal normal. La auscultación cardiaca mostraba tonos arrítmicos y la respiratoria era normal.

La analítica de sangre mostraba glucosa $32 \mathrm{mgr} / \mathrm{dl}$; creatinina $8,75 \mathrm{mg} / \mathrm{dl}(\mathrm{FG}<30) ; \mathrm{Cl} 97 \mathrm{mmol} / \mathrm{l}, \mathrm{Na} 140 \mathrm{mmol} / \mathrm{l} ; \mathrm{K} 4,68$ $\mathrm{mmol} / \mathrm{L}$, Leucocitos 24.000 (N 70, L21, M2), Hb 11,9 gr/dl; Hto 39,9\%; VCM 86; HCM 26, Plaquetas 448.000 TP 75\%. Lactato $32,42 \mathrm{mmol} / 1$. Biomarcadores cardiacos negativos. Anion GAP 40. La gasometría venosa mostraba $\mathrm{pH}$ 6,66; $\mathrm{HCO} 4,20 \mathrm{pCO}_{2} 36$. No presentaba cuerpos cetónicos en orina. La radiografía de tórax era normal y el ECG mostraba una AC x FA con FVM de 110x'. Se realizó un TAC abdominal sin contraste que no mostró alteraciones.

Con el diagnóstico sindrómico de acidosis metabólica normoclorémica con anion gap y lactatos elevados, fracaso renal agudo e hipoglucemia se inicio sueroterapia y aporte de bicarbonato en perfusión endovenosa, indicando hemodiálisis que la paciente no toleró siendo exitus

La acidosis láctica por metformina es una condición metabólica poco frecuente pero de alta mortalidad. El mecanismo exacto por el que la inducen es incierto pero parece estar mediada por la disminución de la actividad de la piruvato-deshidrogenasa y el transporte mitocondrial de los agentes óxido-reducción aumentando el metabolismo anaerobio y acumulando los precursores para el ciclo de Krebs. Esto produce un aumento del paso de piruvato a lactato y por tanto de ácido láctico (2). Su incidencia se estima entre 2-9 casos por 100.000 habitantes y año con una mortalidad en torno al 40\% (3). Se distinguen tres situaciones con diferentes pronósticos: en el primer supuesto la metformina parece ser el causante por una sobredosis siendo el pronóstico bueno; en el segundo caso es producida más por coincidencia que por causalidad pudiendo ser inducida por un fallo orgánico subyacente. En el tercer caso hay una causa de acidosis láctica que empeora por un desencadenante que lleva al acúmulo de metformina. Los dos últimos supuestos son los de peor pronóstico con mortalidades entre $40-50 \%$ porque son los factores condicionantes los que predisponen a la mortalidad y las que necesitan de una hemofiltración $(4,5)$. Es por ello que está contraindicada con creatininas mayores de $1,7 \mathrm{mg} / \mathrm{dl}$, en situaciones de hipoxia-hipoperfusión y en relación con contrastes yodados. Habitualmente los pacientes presentan signos de shock, hipoxia tisular, fallo hepático o renal (6). En nuestro caso la presencia de una insuficiencia renal previa -que se refirió a posteriori- junto con el cuadro de diarreas que presentaba de forma crónica (posiblemente también mediado por la metformina) son los detonantes para la caída en acidosis láctica. De las causas de acidosis metabólica con anion gap elevado la cetoacidosis diabética quedaba descartada por motivos obvios, así como la intoxicación por salicilatos, etilenglicol, metanol o cianuro por la historia clínica. Tampoco presentaba datos de sepsis ni de rabdomiolisis y sólo se planteó la posibilidad de una isquemia intestinal como desencadenante por lo que se realizó un TAC abdominal que fue normal. El único posible desencadenante eran los fármacos y de los que la paciente tomaba sólo la metformina tenía descrito dicho efecto adverso.

En resumen, siendo al metformina un fármaco ampliamente utilizado ha de ser evitada en situaciones de fracaso renal crónico o agudización del mismo y en pacientes que presenten factores concomitantes que favorezcan su desarrollo. Los pacientes en situación de acidosis láctica y fracaso renal se benefician de tratamiento con bicarbonato y hemodiálisis a pesar de lo que la tasa de mortalidad es elevada (6).

\section{P. J. Marchena Yglesias, I. García González, N. Rico Villoria, P. Castellanos Llauger, A. Ricart Conesa}

Servicio de Urgencias. Medicina Interna. Hospital General de L'Hospitalet. L'Hospitalet de Llobregat. Barcelona 